\title{
Study of Knowledge Internalization and Improving Ways of Theme-based Flipped Teaching Under the Perspective of Cognitive Load
}

\author{
Zhang Tongguang, Gao Jianjun \\ Heilongjiang University of Technology, Jixi city
}

Keywords: Cognitive load, Theme-based, Flipped teaching, Knowledge internalization.

\begin{abstract}
The Study of Knowledge Internalization and Improving Ways of Theme-based Flipped Teaching Under the Perspective of Cognitive Load is discussed from the perspective of students' knowledge cognition and it analyzes the process of students' cognition on knowledge learning and knowledge internalization, besides, taking that process as the pointcut and taking the knowledge internalization as the fundamental to build professional thematic learning unit system, furthermore, on the basis of recognizing and mastering teaching knowledge internalization, build the thematic unit-typed flipped teaching improving ways and encourage students to find the rebuild their obtained information so that to promote the students to explore and study actively the problems to be solved, which turns the learning to one activity full of meaning and constructiveness in the process of problem discussion and solving.
\end{abstract}

\section{Introduction}

The Ministry of Education, in the issued Ten-year Development Plan of Educational Informatization(2011-2020), has pointed out that 'taking the ability as the most important', which requires optimizing knowledge structure, enriching social practice and intensifying ability cultivation, puts efforts into improving students' learning ability, practical ability and innovative ability, educates students to learn intellectual skills, learn the ability of practice and thought learn the living and survival ability, learn the ability of being a man and doing things, besides, promotes students to adapt to the society and create the future initiatively. Therefore, it is particularly important to innovate talents cultivation, improve the quality of talents cultivation in the process of teaching practice and take the exploration and study of theme-based flipped teaching under the perspective of cognitive load.

\section{Significance of the Study of Knowledge Internalization and Improving Ways of Theme-based Flipped Teaching Under the Perspective of Cognitive Load}

This study takes students' cognitive load and knowledge internalization as the pointcut and the content is about the process of students' acceptance and internalization of knowledge, besides, design the major according to the task to form a number of targeted theme-based teaching unit, furthermore, drive students to get the in-depth understanding of the subject through the data collection and arrangement, problem solving and active exploration, encourage students to discover and reconstruct their obtained information and promote students to explore and solve problems so as to make them get time to communicate with teachers and also make learning be a constructive activity. In the process of transforming it into personal knowledge, it can help students construct their own understanding frame of knowledge so that they can know how to learn and then achieve the integration of subject knowledge, the integration of learning and society and the integration of learning and students, furthermore, it can also cultivate students' advanced thinking ability and problem solving ability and then promote the learning transfer. The study advocates the integration of courses in the form of subject, so that the students can understand theme-based problems from a 
broad perspective, experience the knowledge formation process, cultivate thinking ability and form a dialectical exploration attitude. It not only emphasizes students' active exploration, also emphasizes students' experience of knowledge formation process so as to form the good cognition and exploration quality, improve problem-solving ability and self-education ability.

This study is conducive to updating teachers' concept, advancing the reform of classroom teaching methods and means, highlighting the cultivation of independent learning ability and problem-solving ability. Focus on the students' innovative thinking cultivation and build the new pattern of teaching mode taking the ability cultivating as the center and the activity organizing as the carrier to promote students to participate in the teaching process as the main body, besides, attach importance to the students' all-round development, truly practice the students-centered education concept and give full play to students' initiative, apart from that, take it as the starting point and foothold of all teaching work that promoting students' growth.

\section{Knowledge Internalization of Theme-based Flipped Teaching Under the Perspective of Cognitive Load}

Students need to complete definite quantity of accumulation of targeted knowledge or activity operating skills in the course of learning, furthermore, keep them in memory for a long time, therefore, the load that is added to students' cognitive system appears when dealing with the specific target tasks. Cognitive load is added to the student's own cognitive system when dealing with the specific problems and it consists of the interactive causal dimensions between reflection matters and students features and the evaluation dimensions reflecting the measurable concepts of mental load, mental effort and performance, besides, it also includes internal cognitive load, external cognitive load, and relevant cognitive load.

The internal cognitive load refers to the generated load when students deal with the information content of cognitive matter itself and take the mutual cognition and it is influenced by the characteristics and complexity of the exploratory matters, besides, the experience range in the minds of the students who are skilled in mastering the past knowledge is matched or cognitively matched to the knowledge to be obtained, the students will have the small cognitive load. Therefore, the degree of the internal cognitive load mainly depends on the cognitive matter itself and students' previous related knowledge and experience background. So, the theme-based flipped teaching based on the internal cognitive load should be based on students' preliminary knowledge and the intervention of theme-based tasks, and then a certain degree of recognition and experience range will generate in the student's mind, furthermore, in the later period of knowledge teaching and exploration, the teachers can make students accept and understand more quickly, thus reduce students' cognitive load and enhance students' sense of learning accomplishment and self-perception.

The external cognitive load refers to that in the learning process, it is difficult for students to match the tasks that they are exploring with their previous experience, or students have no recognition, or the things they have explored are too complex to accept, or the teachers' teaching design or content organization is unreasonable, as a result, it leads to students' rejection of the scheme, which all will bring additional burdens to students, lead to additional cognitive understanding and crowding out cognitive resources. Therefore, the external cognitive load is bad for the students. The theme-based flipped teaching based on the external cognitive load should fully recognize the students' knowledge acceptance pattern and existing level, besides, through combining with the students' characteristics, adopt reasonable teaching methods and scientific design to increase the compatibility and reduce the students' memory load, so that the students can be better and faster to obtain and automatically arrange knowledge.

Relevant cognitive load refers to the load that can effectively promote students' better construction and understanding of cognitive schema and it is the effective cognitive load promoting students' learning. It generates based on the students' digesting ability(ability of achieving mastery through a comprehensive study of the subject) or quality application ability and it is the flexible load related to the degree of students' effort, besides, it is the cognitive load that is latent and subtle. 
Therefore, in the process of knowledge internalization, the theme-based flipped teaching based on relevant cognitive load should pay attention to students' quality education and the promotion of innovation ability, strengthen the ability of critical thinking and cognition, help students construct a rich schema, reduce their load and put the remaining cognitive resources into substantive cognitive activities to improve students' learning efficiency.

In the process of teaching design, the theme of the knowledge internalization of the theme-based flipped teaching based on cognitive load is to reduce its own complexity and external exclusivity of students' learning content, reduce the burden and interference of external cognitive load, improve the integration degree of relevant cognitive load and realize the understanding of schema and the automatic immersion of rules.

\section{Study of Knowledge Internalization and Improving Ways of Theme-based Flipped Teaching Under the Perspective of Cognitive Load}

The existing cognitive structure of students determines whether the new knowledge can be successfully accepted, for new learning materials, the existing relevant knowledge in the cognitive structure of the students plays a guiding and reinforcing role, thus reduces the number of students' processing information and controls the internal cognitive load. Therefore, teachers should firstly understand the students' previous cognitive structure and find the fixed points of the knowledge structure, however the knowledge element needed by college students is of overlarge number, besides, too much information will cause the increase of cognitive load significantly and affect students' memory efficiency, as a result, the knowledge schema can not be clearly constructed. Effective learning requires the active application of working memory to understanding and processing learning objects and storing the incoming information in long-time memory in encoded form.However, the capacity of working memory is limited, and once the total amount of information received exceeds the capacity of working memory, it will obstruct the information processing and then lead to the poor efficiency or even the failure of learning activities. Therefore, in order to gain effective learning, it is necessary to recognize and grasp the cognitive load of students, find a way to rapidly relieve cognitive load in limited time and space and effectively use the limited resources of working memory to reduce the irrelevant cognitive load, besides, make the amount of intellectual activity that is required to be applied to working memory does not exceed the total amount of resources owned by working memory and avoid the overlarge cognitive load caused by insufficient resource allocation.

The so-called theme refers to the realistic core topic or problem with effective load cognition and professional application cognition and it is often the focus of specialized courses or professional tasks, besides, it provides organizational center for curriculum development and stimulating students' learning. The topics include their own attributes and the relationships, principles, theories and norms of the matters, besides, a theme can be broken down into several problems, which can be concretely translated into operable tasks or activities. The theme design based on students' cognitive load is the work of research and development, exploitation and generation about the students' age characteristics, cognitive level, professional knowledge, professional goals etc. and it takes the solving of a certain problem as the intended purpose, besides, it takes the systematic construction of the students' previous knowledge cognition and the knowledge related to task activities from point to line, from line to surface, from surface to body so as to promote students to form systematic and structured thinking in the comprehensive practical activities with 'theme' as the core besides, gradually enrich and improve the knowledge system and cognitive structure, finally turn the learning process to a purposeful, planned, open, flexible, comprehensive and practical process.

The utilization and construction of cognitive environment can affect students' external cognitive load and correlated cognitive load. The teaching of colleges and universities often make use of the physical matters, scene, tools, practical training and simulation activities to present the knowledge to students, only rational organization of these resources can increase students' cognition, reduce external cognitive load, deepen the consistency and compatibility of internal cognitive load so that to make the cognitive load change its previous experience domain and knowledge domain and then 
promote itself from perceptual cognition to rational cognition, finally improve the learning efficiency. Meanwhile, the harmonious and positive inquiry-based cognitive environment can also help to stimulate students' interest and enhance their integration degree, furthermore, increase the relevant cognitive load in the process of mutual inquiry and enlightenment so that to improve the learning efficiency.

The presentation mode of knowledge reflects directly the strengths and weaknesses of teaching. In the course of teaching, in order to improve efficiency, it is necessary to provide proper teaching design and operation method to avoid unnecessary external cognitive load; To appropriately increase the relevant cognitive load to keep the knowledge schema in the long-term memory. Short-term memory has the characteristics of forgetting, therefore, in the process of teaching, efforts should be made to deepen the impression of knowledge in the minds to help students reduce knowledge forgetting, consolidate the accumulation of knowledge and cultivate gradually the students' cognitive load competence ability. Effective presentation pattern of teaching behavior should reflect the interestingness, novelty, variability and diversity.

Self-directed learning refers to the learning mode of determining and completing specific learning goals by students themselves under the guidance of teachers according to their own conditions and needs, the features of cognitive load and the teaching goals. Self-directed learning is the incremental and dynamic process and the students' autonomy degree is a continuum, besides, the students' mastery and understanding of relevant issues involves the stimulation of their own relevant knowledge and experience, therefore, if the study content has nothing to do with the students' existing knowledge and experience, then it is difficult to be recognized. Therefore, the existing cognitive structure provides basis for students to choose their learning content and in the process of theme-based flipped teaching, the students have to take the in-depth study according to their own conditions and thematic tasks, carefully explore the relevant knowledge and thematic content to form the problem cognition, only in which way, can they get better understanding and grasping of the inquiry content.

Group collaborative theme-based inquiry is the cooperated study by students in group form around a topic in their study activities and the task of the teacher is to lead, control and help the students, so that the students can get inspiration and perception through the group collaboration and also make the emotions, attitudes and values collide in the inquiry, furthermore, get cultivation and improvement, in which way, it can influence students' thinking consciousness, make their learning autonomy, initiative and cooperativeness get full play, besides, be conducive to the cultivation of students' innovative thinking and innovation ability and make the problems of inquiry get the integration, understanding and mastery.

Report sharing is the high-level summary and generalization of students' self-knowledge as well as the comparison and relationship between students, besides, by the report sharing, the students, on one hand, can systematically summarize the knowledge and experience gained from learning and comprehension to make the scattered and fragmented knowledge get systematic and integrated arrangement; On the other hand, by comparing the reports and sharing of different students, the students can further grasp the relation between knowledge points to gain new inspirations, broaden their own cognition, measure their own understanding and mastery degree of the problem as well as the lack of self-awareness.

Interactive integration refers to that the teachers take multiple views of detailed analysis and summary in terms of students' understanding and cognition, besides, in view of the entry point, analysis angle, position and viewpoint of students' problems, on one hand, take further analysis and discussion with the students to clarify the essence of the problem and make the students reinforce their learned knowledge and expand the horizontal range; On the other hand, further analysis of students' thinking points and understanding points can also enable students to further cognize themselves, achieve dynamic adaptation and reduce the external cognitive load.

The theme-based flipped teaching under the perspective of cognitive load needs to take evaluation of this method based on the cognitive coincidence, association and symbiosis from the perspective of internal, external and relevant cognitive load so find out the deficiency and problems, 
so that to lay the foundation for the further development and perfection of theme-based flipped teaching under the perspective of cognitive load.

\section{Acknowledgements}

The content of this paper is the scientific research subject of the $13^{\text {th }}$ five-year plan put by Higher Education Academy of Heilongjiang province, namely the Study Result of Knowledge Internalization and Improving Ways of Theme-based Flipped Teaching Under the Perspective of Cognitive Load(subject code:16G493)

\section{References}

[1] Song Yanling, Meng Zhaopeng, Yan Yajuan, Inquiry of Flipped Classroom from the Perspective of Cognitive Load--Analysis of the Typical Pattern of Flipped Classroom, Journal of Distance Education[J], 2014.1.

[2] Zhang Dongmei, Lu Haidong, Zu Yatong, Effect of Knowledge Inversion Under the Perspective of Cognitive Load, Advances In Psychological Science[J], 2016.4.

[3] Liu Jiaojiao, Inquiry of Flipped Classroom from the Perspective of Cognitive Load--Analysis of the Typical Pattern of Flipped Classroom, West Leather[J], 2016.14. 